\title{
Cationic Palladium-Catalyzed Stereoselective Glycosylation with Glycosyl Trichloroacetimidates
}

Jaemoon Yang, Colleen Cooper-Vanosdell, Enoch A. Mensah, and Hien M. Nguyen*

Department of Chemistry and Biochemistry, Montana State University, Bozeman, MT 59717

\section{Table of Content}

Methods and Reagents

General Glycosylation Procedure

Analytical Data of All Compounds

${ }^{1} \mathrm{H}$ NMR and ${ }^{13} \mathrm{C}$ spectra of All Compounds
Page 1

Page 2

Page 2-13

Page 14-63

\section{Supporting Information}

Methods and Reagents. All reactions were performed in oven-dried Schlenk flasks fitted with glass stoppers under a positive pressure of argon. Organic solutions were concentrated by rotary evaporation below $40{ }^{\circ} \mathrm{C}$ at 25 torr. Analytical thin-layer chromatography (TLC) was routinely used to monitor the progress of the reactions and performed using precoated glass plates with 230-400 mesh silica gel impregnated with a fluorescent indicator (250 $\mathrm{nm})$. Visualization was achieved using UV light, iodine, or ceric ammonium molybdate. Flash chromatography was performed and employed 230-400 mesh silica gel. Dichloromethane and toluene were distilled from calcium hydride under an argon atmosphere at 760 torr. All other chemicals were obtained from commercial vendors and used without further purification.

Instrumentation. All proton $\left({ }^{1} \mathrm{H}\right)$ NMR spectra were recored at 300 or $500 \mathrm{MHz}$ and all carbon $\left({ }^{13} \mathrm{C}\right) \mathrm{NMR}$ spectra were recorded at $75 \mathrm{MHz}$ or $125 \mathrm{MHz}$. Chemical shifts are expressed in parts per million ( $\delta$ scale) downfield from tetramethylsilane and are referenced to the residual proton in the NMR solvent $\left(\mathrm{CDCl}_{3}: \delta 7.26 \mathrm{ppm}, \delta 77.23 \mathrm{ppm} ; \mathrm{C}_{6} \mathrm{D}_{6}: \delta 7.16 \mathrm{ppm}, \delta 128.39 \mathrm{ppm}\right)$. Data are presented as follows: chemical shift, multiplicity $(\mathrm{s}=$ singlet, $\mathrm{d}=$ doublet, $\mathrm{t}=$ triplet, $\mathrm{q}=$ quartet, $\mathrm{m}=$ multiplet, and bs = broad singlet), integration, and coupling constant in hertz (Hz). 


\section{General glycosylation procedure with $\mathrm{Pd}\left(\mathrm{CH}_{3} \mathrm{CN}\right)_{4}\left(\mathrm{BF}_{4}\right)_{2}$}

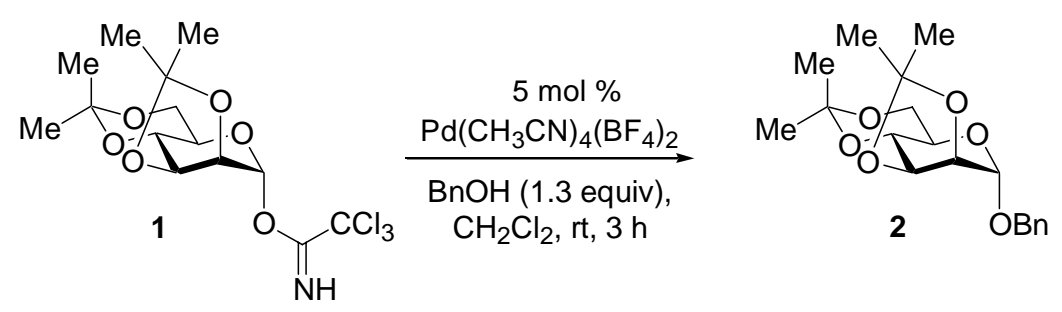

A $10 \mathrm{~mL}$ oven-dried schlenk flask was charged with $\alpha$-D-mannopyranosyl trichloroacetimidate 1 (81 mg, $0.20 \mathrm{mmol}, 1$ equiv), benzyl alcohol (27 $\mu \mathrm{L}, 0.26 \mathrm{mmol}, 1.3$ equiv), and $\mathrm{CH}_{2} \mathrm{Cl}_{2}(1 \mathrm{~mL}) . \mathrm{Pd}\left(\mathrm{CH}_{3} \mathrm{CN}\right)_{4}\left(\mathrm{BF}_{4}\right)_{2}(4.4 \mathrm{mg}, 0.01 \mathrm{mmol}, 5 \mathrm{~mol} \%)$ was then added to the solution . The resulting mixture was stirred at $25{ }^{\circ} \mathrm{C}$ for $3 \mathrm{~h}$, diluted with benzene $(1 \mathrm{~mL})$, and purified by silica gel flash chromatography (6/1, hexane/ethyl acetate) to give the desired product 2 (59.2 $\mathrm{mg}, 85 \%)$ as a white solid.

MP: $48-49{ }^{\circ} \mathrm{C}$

${ }^{1} \mathbf{H}$ NMR (CDCl 3 , 500 MHz): $\delta=7.35-7.26$ (m, 5H), 5.05 (s, 1H), 4.77 (dd, $J=6.0,3.5 \mathrm{~Hz}$, $1 \mathrm{H}), 4.64$ (d, $J=6.0 \mathrm{~Hz}, 1 \mathrm{H}), 4.62$ (d, $J=11.5 \mathrm{~Hz}, 1 \mathrm{H}) 4.47$ (d, $J=11.5 \mathrm{~Hz}, 1 \mathrm{H}), 4.39$ (ddd, $J=$ 7.5, 6.5, $4.5 \mathrm{~Hz}, 1 \mathrm{H}$ ), 4.09 (dd, $J=9.0,6.5 \mathrm{~Hz}, 1 \mathrm{H}$ ), 3.98-3.94 (m, 2H), 1.44 (s, 3H), 1.43 (s, 3H), 1.37 (s, 3H), 1.30 (s, 3H).

${ }^{13} \mathbf{C}$ NMR (CDCl 3,125 MHz): $\delta=137.3,128.5,128.0,127.8,112.6,109.2,105.6,85.1,80.4$, 76.7, 73.1, 69.1, 66.9, 26.9, 25.8, 25.2, 24.5 .

IR (film, $\mathbf{c m}^{-1}$ ): $v=$ 3031, 2986, 2937, 1455, 1372, 1210, 1077, 1021, 699.

HRMS (ESI): calc. for $\mathrm{C}_{19} \mathrm{H}_{26} \mathrm{O}_{6} \mathrm{Na}$ [M+Na] 373.1622; found: 373.1694.

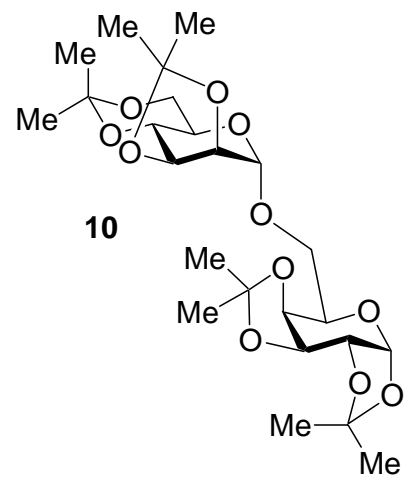

${ }^{1} \mathrm{H}$ and ${ }^{13} \mathrm{C}$ NMR of disaccharide $\mathbf{1 0}$ has been reported. ${ }^{\mathrm{i}}$

${ }^{1} \mathbf{H}$ NMR (CDCl, 500 MHz): $\delta=5.50$ (d, $\left.J=5.0 \mathrm{~Hz}, 1 \mathrm{H}\right), 4.99$ (s, 1H), 4.75 (dd, $J=6.0,3.5$ $\mathrm{Hz}, 1 \mathrm{H}), 4.60$ (d, $J=6.0 \mathrm{~Hz}, 1 \mathrm{H}), 4.56$ (dd, $J=8.0,2.5 \mathrm{~Hz}, 1 \mathrm{H}), 4.34$ (ddd, $J=8.0,6.0,4.0 \mathrm{~Hz}$, $1 \mathrm{H}$ ), 4.28 (dd, $J=5.5,2.5 \mathrm{~Hz}$ ), 4.17 (dd, $J=8.0,2.0 \mathrm{~Hz}, 1 \mathrm{H}$ ), 4.06 (dd, $J=8.5,6.0 \mathrm{~Hz}, 1 \mathrm{H}$ ), 3.99 (d, $J=9.0,4.5 \mathrm{~Hz}, 1 \mathrm{H}), 3.93$ (dd, $J=8.5,4.0 \mathrm{~Hz}, 1 \mathrm{H}), 3.91$ (dd, $J=7.0,2.0 \mathrm{~Hz}, 1 \mathrm{H}$ ), 3.70 (dd, $J=10.5,6.5 \mathrm{~Hz}, 1 \mathrm{H}), 3.60$ (dd, $J=10.5,7.0 \mathrm{~Hz}, 1 \mathrm{H}), 1.50$ (s, 3H), 1.42 (s, 3H), 1.41 (s, 3H), 1.40 (s, 3H), 1.34 (s, 3H), 1.30 (s, 3H), 1.29 (s, 3H), 1.28 (s, 3H). ${ }^{1} \mathrm{H}$ NMR matches with the literature report.

${ }^{13} \mathbf{C}$ NMR (CDCl 3 , 125 MHz): $\delta=112.6,109.4,109.3,108.6,106.7,96.3,85.0,80.4,79.5$, 73.0, 70.9, 70.6, 70.5, 66.9, 66.1, 66.0, 26.9, 26.0, 25.9, 25.8, 25.2, 24.9, 24.6, 24.5. ${ }^{13} \mathrm{C}$ NMR matches with the literature report.

IR (film, $\mathbf{c m}^{-\mathbf{1}}$ ): $v=2987,2937,1373,1256,1211,1165,1069,1001,846$. 


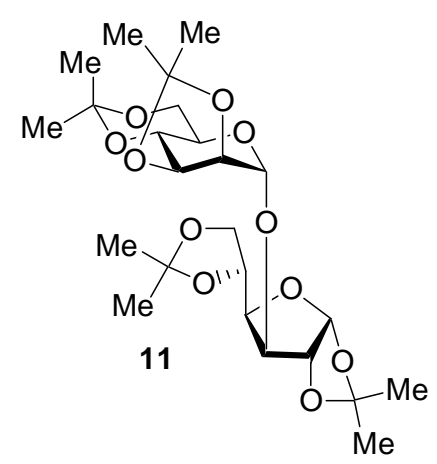

${ }^{1} \mathrm{H}$ and ${ }^{13} \mathrm{C}$ NMR of disaccharide $\mathbf{1 1}$ has been reported. ${ }^{\mathrm{i}}$

${ }^{1} \mathbf{H}$ NMR (CDCl 3,500 MHz): $\delta=5.79(\mathrm{~d}, J=3.0 \mathrm{~Hz}, 1 \mathrm{H}$ ), 5.19 (s, $1 \mathrm{H}), 4.73$ (dd, $J=6.0,3.5$ $\mathrm{Hz}, 1 \mathrm{H}$ ), 4.58 (d, $J=5.5 \mathrm{~Hz}, 1 \mathrm{H}$ ), 4.53 (d, $J=3.5 \mathrm{~Hz}, 1 \mathrm{H}$ ), 4.37 (dd, $J=110,6.0 \mathrm{~Hz}, 1 \mathrm{H}), 4.18$ (m, 2H), 4.09-3.99 (m, 4H), 3.95-3.92 (m, 2H), 1.45 (s, 3H), 1.42 (s, 6H), 1.37 (s, 3H), 1.34 (s, $3 \mathrm{H}), 1.30$ (s, 3H), 1.28 (s, 3H), 1.27 (s, 3H). ${ }^{1} \mathrm{H}$ NMR matches with the literature report.

${ }^{13} \mathbf{C}$ NMR (CDCl 3 , 125 MHz): $\delta=112.7,111.9,109.2,109.1,107.7,105.1,84.9,83.6,80.8$, 80.7, 79.3, 73.1, 72.3, 67.4, 66.6, 26.9, 26.8, 26.2, 25.8, 25.3, 25.1, 24.5. ${ }^{13} \mathrm{C}$ NMR matches with the literature report.

IR (film, $\mathbf{c m}^{-1}$ ): $v$ = 3365, 3194, 2988, 2938, 1455, 1374, 1213, 1163, 1115, 1078, 1020, 845.

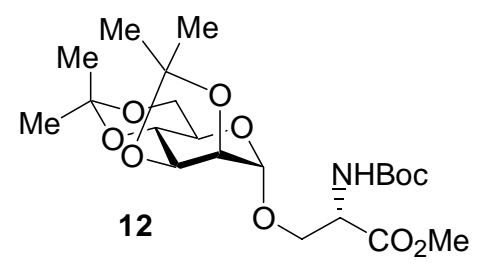

${ }^{1} \mathbf{H}$ NMR $\left(\mathbf{C D C l}_{3}, \mathbf{5 0 0} \mathbf{~ M H z}\right): \delta=5.43(\mathrm{~d}, J=9.0 \mathrm{~Hz}, 1 \mathrm{H}, \mathrm{NH}), 4.91$ (s, $\left.1 \mathrm{H}\right), 4.75$ (dd, $J=6.0$, $3.5 \mathrm{~Hz}, 1 \mathrm{H}), 4.53(\mathrm{~d}, J=6.0 \mathrm{~Hz}, 1 \mathrm{H}), 4.38-4.36(\mathrm{~m}, 1 \mathrm{H}), 4.35$ (dd, $J=11.5,6.5 \mathrm{~Hz}, 1 \mathrm{H}), 4.09$ (dd, $J=8.5,6.5 \mathrm{~Hz}, 1 \mathrm{H}$ ), 4.01 (dd, $J=8.5,4.5 \mathrm{~Hz}, 1 \mathrm{H}$ ), 3.91-3.87 (m, 2H), 3.80 (dd, $J=10.5$, $3.0 \mathrm{~Hz}, 1 \mathrm{H}$ ), 3.74 (s, 3H), 1.44 (s, 9H), 1.43 (s, 6H), 1.36 (s, 3H), 1.29 (s, 3H).

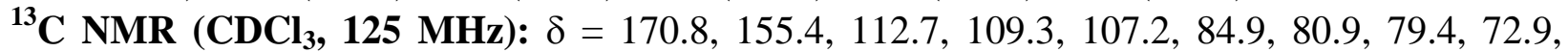
68.7, 67.0, 53.9, 52.5, 29.7, 28.3, 26.8, 25.8, 25.2, 24.5.

IR (film, $\mathbf{~ c m}^{-1}$ ): $v=2983,2936,1721,1507,1370,1253,1211,1163,1117,1066,1028,827$.

HRMS (ESI): calc. for $\mathrm{C}_{21} \mathrm{H}_{35} \mathrm{NO}_{10} \mathrm{Na}$ [M+Na] 484.2153; found: 484.2159 .

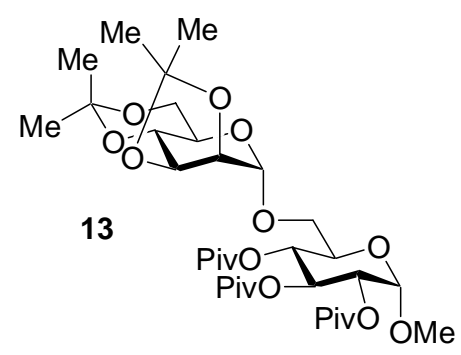

${ }^{1} \mathbf{H}$ NMR (CDCl $\left.3,500 \mathbf{~ M H z}\right): \delta=5.49(\mathrm{t}, J=9.5 \mathrm{~Hz}, 1 \mathrm{H}), 5.00(\mathrm{~s}, 1 \mathrm{H}), 4.83(\mathrm{~d}, J=3.5 \mathrm{~Hz}$, $1 \mathrm{H}), 4.72$ (dd, $J=5.5,3.5 \mathrm{~Hz}, 1 \mathrm{H}), 4.66(\mathrm{dd}, J=10.5,4.0 \mathrm{~Hz}, 1 \mathrm{H}), 4.49(\mathrm{t}, J=12.0 \mathrm{~Hz}, 1 \mathrm{H}$ ), $4.46(\mathrm{~d}, J=6.0 \mathrm{~Hz}, 1 \mathrm{H}), 4.31-4.27(\mathrm{~m}, 1 \mathrm{H}), 4.06-3.95(\mathrm{~m}, 4 \mathrm{H}), 3.83-3.80(\mathrm{~m}, 1 \mathrm{H}), 3.57(\mathrm{t}, J=$ 
$9.5 \mathrm{~Hz}, 1 \mathrm{H}), 3.32$ (s, 3H), 1.39 (s, 3H), 1.37 (s, 3H), 1.34 (s, 3H), 1.24 (s, 3H), 1.19 (s, 9H), 1.319 (s, 9H), 1.14 (s, 9H).

${ }^{13} \mathbf{C}$ NMR (CDCl 3 , 125 MHz): $\delta=177.9,177.8,176.9,112.7,109.2,109,1,96.3,85.3,81.1$, 79.4, 73.0, 71.4, 71.1, 68.3, 66.7, 63.0, 55.3, 33.9, 33.8, 38.7, 27.2, 27.1, 26.9, 26.6, 25.8, 25.4, 24.6.

IR (film, $\mathbf{c m}^{-1}$ ): $v=2978,2937,1734,1481,1371,1281,1159,1065,1037,977$.

HRMS (ESI): calc. for $\mathrm{C}_{34} \mathrm{H}_{56} \mathrm{O}_{14} \mathrm{Na}$ [M+Na] 711.3568; found: 711.3609 .

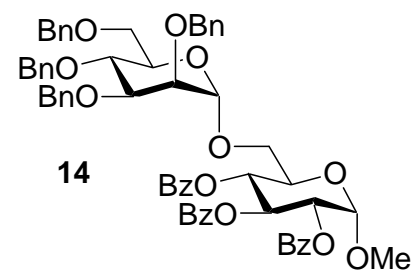

${ }^{1} \mathrm{H}$ and ${ }^{13} \mathrm{C}$ NMR of disaccharide $\mathbf{1 4}$ has been reported. ${ }^{\mathrm{ii}}$

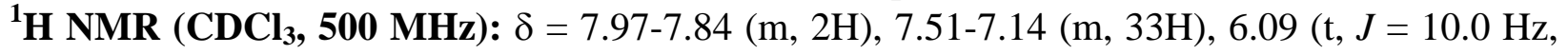
1H), 5.54 (t, $J=10.0 \mathrm{~Hz}, 1 \mathrm{H}), 5.21$ (dd, $J=10.0,3.5 \mathrm{~Hz}, 1 \mathrm{H}), 5.15$ (d, $J=3.5 \mathrm{~Hz}, 1 \mathrm{H}), 4.90$ (d, $J=1.0 \mathrm{~Hz}, 1 \mathrm{H}), 4.85(\mathrm{~d}, J=10.5 \mathrm{~Hz}, 1 \mathrm{H}), 4.70(\mathrm{~d}, J=12.5 \mathrm{~Hz}, 1 \mathrm{H}), 4.66(\mathrm{~d}, J=12.5 \mathrm{~Hz}, 1 \mathrm{H})$, $4.53(\mathrm{~d}, J=12.0 \mathrm{~Hz}, 1 \mathrm{H}), 4.50-4.42(\mathrm{~m}, 3 \mathrm{H}), 4.37$ (d, $J=12.0 \mathrm{~Hz}, 1 \mathrm{H}), 4.15-4.11(\mathrm{~m}, 1 \mathrm{H}), 3.93$ (t, $J=9.0 \mathrm{~Hz}, 1 \mathrm{H}), 3.88-3.83$ (m, 3H), 3.68-3.67 (m, 1H), 3.64-3.58 (m, 3H), 3.53-3.50 (m, 1H), 3.37 (s, 3H). ${ }^{1} \mathrm{H}$ NMR matches with the literature report.

${ }^{13} \mathbf{C}$ NMR (CDCl 3 , 125 MHz): $\delta=165.8,165.1,138.7,138.6,138.4,138.3,133.3,133.2,133.1$, 129.9, 129.6, 129.3, 129.1, 128.6, 128.4, 128.31, 128.3, 128.2, 127.9, 127.8, 127.7, 127.52, 127.5, 127.4, 98.2, 96.9, 79.8, 74.9, 74.8, 74.7, 73.2, 72.5, 72.1, 71.8, 70.5, 69.8, 68.9, 68.1, 66.1, 55.5. ${ }^{13} \mathrm{C}$ NMR matches with the literature report.

IR (film, $\mathbf{c m}^{-\mathbf{1}}$ ): $v=3063,3031,2933,2871,1730,1453,1280,1265,1106,1069,1027,916$, 825.

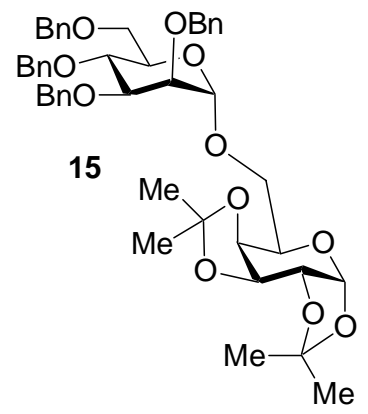

The synthesis of disaccharide $\mathbf{1 5}$ has been reported. ${ }^{\mathrm{iii}}$

${ }^{1} \mathbf{H}$ NMR (C $\left.\mathbf{C}_{\mathbf{6}} \mathbf{D}_{\mathbf{6}}, \mathbf{5 0 0} \mathbf{M H z}\right): \delta=7.46-7.45$ (m, 2H), 7.40-7.36 (m, 4H), 7.31-7.29 (m, 2H), 7257.13 (m, 12H), 5.58 (d, $J=5.0 \mathrm{~Hz}, 1 \mathrm{H}), 5.28$ (s, 1H), 5.03 (d, $J=11.5 \mathrm{~Hz}, 1 \mathrm{H}), 4.72$ (d, $J=12.0$ Hz, 1H), 4.68-4.64 (m, 3H), 4.58 (dd, $J=8.0,2.0$ Hz, 1H), 4.52-4.45 (m, 3H), 4.39 (dd, $J=14.5$, $4.5 \mathrm{~Hz}, 1 \mathrm{H}), 4.32$ (t, $J=6.0 \mathrm{~Hz}, 1 \mathrm{H}), 4.26-4.21$ (m, 3H), 4.17 (ddd, $J=10.5,6.5,3.0 \mathrm{~Hz}, 1 \mathrm{H}$ ), 4.12 (d, $J=8.0 \mathrm{~Hz}, 1 \mathrm{H}), 4.00-3.96$ (m, 2H), 3.89 (dd, $J=11.0,5.0 \mathrm{~Hz}, 1 \mathrm{H}), 3.78$ (d, $J=11.0$ Hz), 1.51 (s, 3H), 1.50 (s, 3H), 1.23 (s, 3H), 1.09 (s, 3H). 
${ }^{13} \mathbf{C}$ NMR (C $\left.\mathbf{C}_{6} \mathbf{D}_{6}, 125 \mathrm{MHz}\right): \delta=139.4,139.2,139.1,138.9,128.3,128.2,128.1,127.72,127.7$, 127.4, 127.3, 127.23, 127.2, 109.2, 108.2, 97.8, 96.6, 80.6. 75.7, 75.3, 74.8, 73.3, 72.9, 72.7, 71.9, 71.3, 71.1, 70.7, 69.6, 66.1, 65.9, 26.0, 24.6, 24.3.

IR (film, $\mathbf{c m}^{-1}$ ): $v=2987,2916,1496,1454,1381,1371,1255,1211,1167,1100,1070,1027$, 1002.

HRMS (ESI): calc. for $\mathrm{C}_{46} \mathrm{H}_{54} \mathrm{O}_{11} \mathrm{Na}$ [M+Na] 805.3558; found: 805.3527.

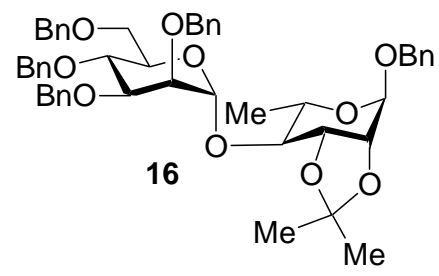

${ }^{1} \mathbf{H}$ NMR (C $\left.\mathbf{C}_{\mathbf{6}} \mathbf{D}_{\mathbf{6}}, 500 \mathrm{MHz}\right): \delta=7.36-7.15$ (m, 25H), 7.40-7.36 (m, 4H), 4.96 (s, 1H), 4.92 (s, $1 \mathrm{H}), 4.87$ (d, $J=11.0 \mathrm{~Hz}, 1 \mathrm{H}), 4.75(\mathrm{~d}, J=12.5 \mathrm{~Hz}, 1 \mathrm{H}), 4.72(\mathrm{~d}, J=12.5 \mathrm{~Hz}, 1 \mathrm{H}), 4.68(\mathrm{~d}, J=$ $12.5 \mathrm{~Hz}, 1 \mathrm{H}), 4.66-4.59(\mathrm{~m}, 3 \mathrm{H}), 4.52$ (d, $J=10.5 \mathrm{~Hz}, 1 \mathrm{H}), 4.47$ (t, $J=11.5 \mathrm{~Hz}, 2 \mathrm{H}), 4.19(\mathrm{t}, J=$ $10.0 \mathrm{~Hz}, 1 \mathrm{H}$ ), 4.08 (d, $J=5.5 \mathrm{~Hz}, 1 \mathrm{H}), 4.01-3.97$ (m, 2H), 3.87 (dd, $J=10.5,3.0 \mathrm{~Hz}, 1 \mathrm{H}$ ), 3.83 (dd, $J=9.5,3.0 \mathrm{~Hz}, 1 \mathrm{H}), 3.72-3.71(\mathrm{~m}, 1 \mathrm{H}), 3.65$ (dd, $J=11.0,1.5 \mathrm{~Hz}, 1 \mathrm{H}), 3.59$ (dq, $J=8.5$, $6.5 \mathrm{~Hz}, 1 \mathrm{H}$ ), 3.32 (dd, $J=10.0,7.5 \mathrm{~Hz}, 1 \mathrm{H}), 1.44$ (s, 3H), 1.23 (s, 3H), 1.02 (d, $J=6.0 \mathrm{~Hz}, 3 \mathrm{H})$.

${ }^{13} \mathbf{C}$ NMR (C $\left.\mathbf{C}_{6} \mathbf{D}_{6}, 125 \mathrm{MHz}\right): \delta=138.8,138.6,138.2,128.5,128.3,128.25,128.2,128.1,128.0$, 127.9, 127.8, 127.6, 127.5, 127.4, 127.3, 109.0, 98.9, 96.1, 80.2, 80.1, 76.0, 75.2, 74.7, 74.2, 73.4, 72.6, 72.3, 71.8. 69.1, 68.7, 65.0, 28.1, 26.3, 17.3.

IR (film, $\mathbf{c m}^{-1}$ ): $v=3029,2984,2934,1496,1454,1380,1242,1219,1139,1093,1051,1027$, 994, 861.

HRMS (ESI): calc. for $\mathrm{C}_{50} \mathrm{H}_{56} \mathrm{O}_{10} \mathrm{Na}[\mathrm{M}+\mathrm{Na}]$ 839.3771; found: 839.3762.

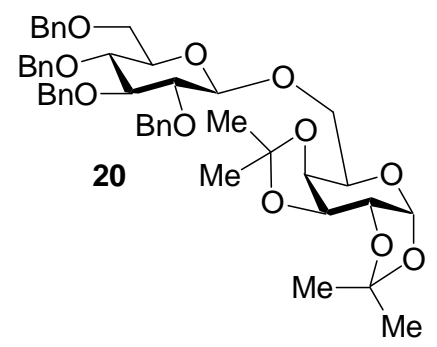

${ }^{1} \mathrm{H}$ and ${ }^{13} \mathrm{C}$ NMR of disaccharide 20 has been reported. ${ }^{\text {iv }}$

$\mathbf{R}_{\mathbf{f}}=0.36$ (hexanes/ethyl acetate, $4 / 1$ )

${ }^{1} \mathbf{H}$ NMR (CDCl 3 , 500 MHz): $\delta=7.44-7.14$ (m, 20H), 5.58 (d, $\left.J=5.0 \mathrm{~Hz}, 1 \mathrm{H}\right), 5.07$ (d, $J=11$ Hz, 1H), 4.97 (d, $J=11 \mathrm{~Hz}, 1 \mathrm{H}), 4.82$ (d, $J=11 \mathrm{~Hz}, 1 \mathrm{H}), 4.79(\mathrm{~d}, J=11 \mathrm{~Hz}, 1 \mathrm{H}), 4.73$ (d, $J=11$ Hz, 1H), 4.65-4.58 (m, 2H), 4.55 (s, 1H), 4.51 (d, $J=11$ Hz, 1H), 4.47 (d, $J=7.5$ Hz, 1H), 4.33$4.31(\mathrm{~m}, 1 \mathrm{H}), 4.25$ (d, $J=8 \mathrm{~Hz}, 1 \mathrm{H}), 4.17$ (dd, $J=10.5,3.5 \mathrm{~Hz}, 1 \mathrm{H}), 4.1(\mathrm{~m}, 1 \mathrm{H}), 3.83-3.68$ (m, 3H), 3.67-3.58 (m, 2H), 3.49-3.45 (m, 2H), 1.51 (s, 3H), 1.46 (s, 3H), 1.32 (2s, 6H). ${ }^{1} \mathrm{H}$ NMR matches with the literature report.

${ }^{13} \mathbf{C}$ NMR (CDCl 3 , 125 MHz): $\delta=138.7,138.2,128.7,128.4,128.2,128.0,127.9,127.7,127.6$, 127.55, 127.49, 109.4, 108.6, 104.4, 96.4, 84.6, 81.6, 77.7, 75.7, 75.0, 74.8, 74.4, 73.5, 71.5, 70.8, 70.5, 69.7, 68.8, 67.4, 26.1, 26.0, 25.1, 24.5. ${ }^{13} \mathrm{C}$ NMR matches with the literature report.

$\boldsymbol{J}\left({ }^{\mathbf{1 3}} \mathbf{C H}\right)=156 \mathrm{~Hz}(104.4 \mathrm{ppm}) ; 179 \mathrm{~Hz}(96.4 \mathrm{ppm})$.

IR (film, $\mathbf{c m}^{-1}$ ): $v=2902,1454,1381,1255,1211$. 


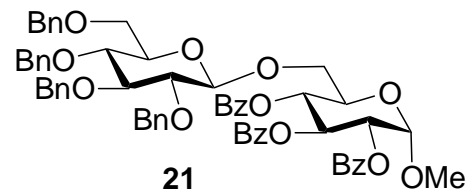

${ }^{1} \mathrm{H}$ and ${ }^{13} \mathrm{C}$ NMR of disaccharide 20 has been reported. ${ }^{\mathrm{v}}$

$\mathbf{R}_{\mathbf{f}}=0.18$ (hexanes/ethyl acetate, 6/1)

${ }^{1} \mathbf{H}$ NMR (CDCl 3,500 MHz): $\delta=7.96$ (d, $\left.J=7.5 \mathrm{~Hz}, 2 \mathrm{H}\right), 7.92$ (d, $\left.J=7.0 \mathrm{~Hz}, 2 \mathrm{H}\right), 7.84$ (d, $J=$ $7.0 \mathrm{~Hz}, 2 \mathrm{H}), 7.51-7.13(\mathrm{~m}, 29 \mathrm{H}), 6.16(\mathrm{t}, J=10 \mathrm{~Hz}, 1 \mathrm{H}), 5.48(\mathrm{t}, J=10 \mathrm{~Hz}, 1 \mathrm{H}), 5.25$ (dd, $J=$ 10, $3.5 \mathrm{~Hz}, 1 \mathrm{H}$ ), 5.20 (d, $J=2.5 \mathrm{~Hz}, 1 \mathrm{H}), 5.05$ (d, $J=10.5 \mathrm{~Hz}, 1 \mathrm{H}), 4.90$ (d, $J=11 \mathrm{~Hz}, 1 \mathrm{H})$, 4.82-4.74 (m, 2H), 4.68 (d, $J=10 \mathrm{~Hz}, 1 \mathrm{H}), 4.53-4.35$ (m, 5H), 4.12 (d, $J=10 \mathrm{~Hz}, 1 \mathrm{H}$ ), 3.80 (dd, $J=10.5,7 \mathrm{~Hz}, 1 \mathrm{H}), 3.64-3.57$ (m, 4H), 3.49-3.40 (m, 2H), 3.37 (s, 3H). ${ }^{1} \mathrm{H}$ NMR matches with the literature report.

${ }^{13}$ C NMR (CDCl 3 , 125 MHz): $\delta=165.84,165.80,165.5,138.6,138.5,138.10,138.07,133.42$, 133.35, 133.08, 130.0, 129.9, 129.7, 129.2, 129.1, 128.9, 128.4, 128.35, 128.26, 128.2, 127.93, 127.87, 127.75, 127.67, 127.57, 127.5, 104.0, 96.8, 84.5, 82.3, 81.7, 77.7, 75.7, 75.0, 74.9, 74.8, 73.4, 72.1, 70.5, 69.9, 69.0, 68.8, 68.6, 55.5. ${ }^{13} \mathrm{C}$ NMR matches with the literature report.

$\mathbf{J}\left({ }^{\mathbf{1 3}} \mathbf{C H}\right)=155 \mathrm{~Hz}(104.0 \mathrm{ppm}) ; 173 \mathrm{~Hz}(96.8 \mathrm{ppm})$.

IR (film, $\mathbf{c m}^{-1}$ ): $v$ 2916, 1730.

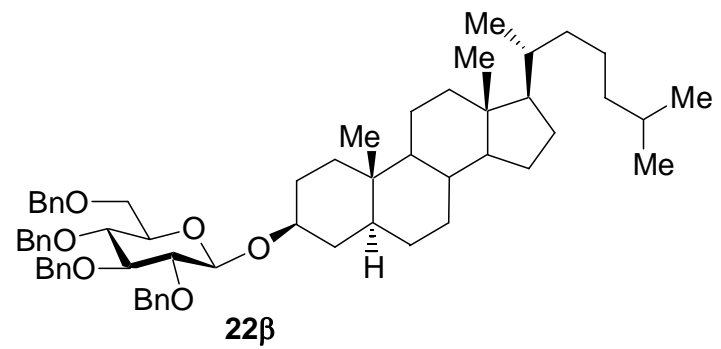

${ }^{1} \mathrm{H}$ and ${ }^{13} \mathrm{C}$ NMR of disaccharide 20 has been reported. ${ }^{\mathrm{vi}}$

$\mathbf{R}_{\mathbf{f}}=0.44$ (hexanes/ethyl acetate, 9/1)

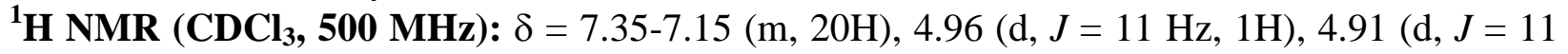
$\mathrm{Hz}, 1 \mathrm{H}), 4.81$ (d, $J=11 \mathrm{~Hz}, 1 \mathrm{H}), 4.77(\mathrm{~d}, J=11 \mathrm{~Hz}, 1 \mathrm{H}), 4.71(\mathrm{~d}, J=11 \mathrm{~Hz}, 1 \mathrm{H}), 4.61-4.51$ (m, 3H), 4.51 (d, $J=8 \mathrm{~Hz}, 1 \mathrm{H}), 3.74$ (d, $J=10.5 \mathrm{~Hz}, 1 \mathrm{H}), 3.65-3.60$ (m, 3H), 3.52 (t, $J=9.0 \mathrm{~Hz}$, $1 \mathrm{H}), 3.46-3.40(\mathrm{~m}, 2 \mathrm{H}), 1.98-0.58(\mathrm{~m}, 47 \mathrm{H}) .{ }^{1} \mathrm{H}$ NMR matches with the literature report.

${ }^{13} \mathbf{C}$ NMR (CDCl 3 , 125 MHz): $\delta=138.7,138.6,138.3,138.2,128.4,128.33,128.26,128.0$, 127.9, 127.73, 127.67, 127.62, 127.55, 127.52, 101.9, 84.9, 82.4, 79.1, 78.1, 75.7, 75.0, 74.8, 73.4, 69.3, 56.5, 56.3, 54.4, 44.8, 42.6, 40.1, 39.5, 37.1, 36.2, 35.8, 35.6, 35.5, 34.8, 32.1, 29.7, 28.8, 28.3, 28.0, 24.2, 23.8, 22.8, 22.6, 21.2, 18.7, 12.3, 12.1. ${ }^{13} \mathrm{C}$ NMR matches with the literature report.

IR (film, $\mathbf{c m}^{-1}$ ): $v=2931,2866,1453,1360$. 


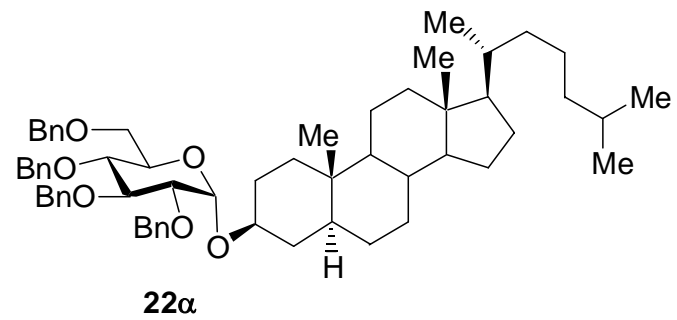

${ }^{1} \mathrm{H}$ and ${ }^{13} \mathrm{C}$ NMR of disaccharide 20 has been reported. ${ }^{\mathrm{vi}}$

$\mathbf{R}_{\mathbf{f}}=0.44$ (hexanes/ethyl acetate, $9 / 1$ )

${ }^{1}$ H NMR (CDCl 3 , 500 MHz): $\delta=7.34-7.11$ (m, 20H), 4.98 (d, $\left.J=11 \mathrm{~Hz}, 1 \mathrm{H}\right), 4.91$ (d, $J=3$ Hz, $1 \mathrm{H}), 4.81$ (d, $J=11 \mathrm{~Hz}, 1 \mathrm{H}), 4.79$ (d, $J=11 \mathrm{~Hz}, 1 \mathrm{H}), 4.74$ (d, $J=12 \mathrm{~Hz}, 1 \mathrm{H}), 4.65$ (d, $J=12$ Hz, 1H), 4.44 (d, $J=11.5$ Hz, 2H), 3.97 (t, $J=9$ Hz, 1H), 3.86 (t, $J=9.5 \mathrm{~Hz}, 1 \mathrm{H}$ ), 3.71 (dd, $J=$ 10, $3 \mathrm{~Hz}, 1 \mathrm{H}), 3.68-3.51$ (m, 4H), 1.96-0.56 (m, 47H).

${ }^{13} \mathbf{C ~ N M R ~ ( C D C l}_{3}, 125$ MHz): $\delta=139.0,138.3,128.4,128.34,128.32,128.1,127.9,127.83$, 127.80, 127.65, 127.61, 127.5, 94.7, 82.1, 80.0, 77.9, 76.3, 75.6, 75.1, 73.4, 73.0, 70.0, 68.7, 56.5, 56.3, 54.4, 45.1, 42.6, 40.0, 39.5, 36.9, 36.2, 35.84, 35.78, 35.7, 35.5, 32.1, 28.7, 28.2, 28.0, 27.4, 24.2, 23.8, 22.8, 22.5, 21.2, 18.7, 12.3, 12.1.

IR (film, $\mathbf{c m}^{-1}$ ): $v=2928,2865,1453$.

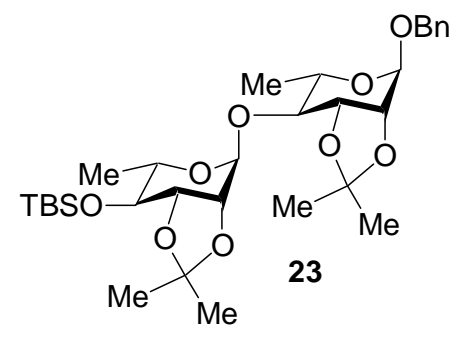

$\mathbf{R}_{\mathbf{f}}=0.42$ (hexanes/ethyl acetate, 6/1)

${ }^{1}$ H NMR (CDCl 3 , 500 MHz): $\delta=7.34-7.29$ (m, 5H), 5.54 (s, 1H), 5.04 (s, 1H), 4.68 (d, $J=11.5$ $\mathrm{Hz}, 1 \mathrm{H}), 4.50(\mathrm{~d}, J=11.5 \mathrm{~Hz}, 1 \mathrm{H}), 4.21(\mathrm{t}, J=6.5 \mathrm{~Hz}, 1 \mathrm{H}), 4.12(\mathrm{~d}, J=5.5 \mathrm{~Hz}, 1 \mathrm{H}), 4.10(\mathrm{~d}, J=$ $5.5 \mathrm{~Hz}, 1 \mathrm{H}), 3.91$ (t, $J=6.0 \mathrm{~Hz}, 1 \mathrm{H}), 3.74-3.68(\mathrm{~m}, 1 \mathrm{H}), 3.59-3.53(\mathrm{~m}, 2 \mathrm{H}), 3.32$ (dd, $J=9.5,7.5$ $\mathrm{Hz}, 1 \mathrm{H}), 1.52$ (s, 3H), 1.50 (s, 3H), 1.32 (s, 3H), 1.30 (s, 3H), 1.26 (d, $J=6 \mathrm{~Hz}, 3 \mathrm{H}), 1.19(\mathrm{~d}, J=$ $6.5 \mathrm{~Hz}, 3 \mathrm{H}), 0.88$ (s, 9H), 0.12 (s, 3H), 0.06 (s, 3H).

${ }^{13} \mathbf{C}$ NMR (CDCl 3 , 125 MHz): $\delta=137.1,128.5,128.2,127.9,109.4,108.7,96.2,95.9,78.9$, 78.6, 76.7, 76.5, 76.1, 75.7, 69.1, 66.4, 64.2, 28.1, 27.9, 26.4, 25.9, 17.9, 17.5, -4.0, -4.8.

IR (film, $\mathbf{c m}^{-1}$ ): $v=2934,1381,1245,1221,1086$.

HRMS (ESI): calc. for $\mathrm{C}_{31} \mathrm{H}_{50} \mathrm{O}_{9} \mathrm{SiNa}(\mathrm{M}+\mathrm{Na})$ 617.3116; found: 617.3097 .

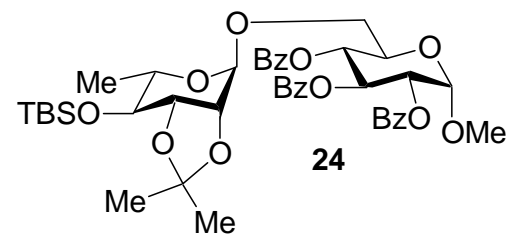

$\mathbf{R}_{\mathbf{f}}=0.49$ (hexanes/ethyl acetate, $2 / 1$ )

${ }^{1} \mathbf{H}$ NMR (CDCl 3,500 MHz): $\delta=7.97-7.92$ (m, 4H), 7.85 (d, $\left.J=8 \mathrm{~Hz}, 2 \mathrm{H}\right), 7.51-7.34$ (m, 7H), 7.27 (t, $J=8 \mathrm{~Hz}, 2 \mathrm{H}), 6.13$ (t, $J=10 \mathrm{~Hz}, 1 \mathrm{H}), 5.56$ (t, $J=10 \mathrm{~Hz}, 1 \mathrm{H}), 5.24$ (dd, $J=10,3.5 \mathrm{~Hz}$, 
1H), 5.21 (d, $J=3.5 \mathrm{~Hz}, 1 \mathrm{H}), 4.91(\mathrm{~s}, 1 \mathrm{H}), 4.22-4.18$ (m, 1H), 4.07 (d, $J=6 \mathrm{~Hz}, 1 \mathrm{H}), 3.86-3.82$ (m, 2H), 3.62 (dd, $J=11.5,5.5 \mathrm{~Hz}, 1 \mathrm{H}), 3.52$ (dd, $J=10,6.5 \mathrm{~Hz}, 1 \mathrm{H}), 3.46$ (s, 3H), 3.25 (dd, $J=$ 9.5, $7.5 \mathrm{~Hz}, 1 \mathrm{H}), 1.47$ (s, 3H), 1.29 (s, 3H), 1.10 (d, J=6 Hz, 3H), 0.87 (s, 9H), 0.11 (s, 3H), 0.04 (s, 3H).

${ }^{13} \mathbf{C}$ NMR (CDCl 3,125 MHz): $\delta=165.82,165.79,165.3,133.5,133.4,133.3,133.0,129.9$, 129.8, 129.7, 128.41, 128.39, 128.3, 108.8, 97.8, 97.0, 78.9, 75.86, 75.80, 72.1, 70.5, 69.7, 68.7, 55.6, 29.7, 28.1, 26.4, 25.9, 17.5, -4.0, -4.9.

IR (film, $\mathbf{c m}^{-1}$ ): $v=2930,1732,1280,1265$.

HRMS (ESI): calc. for $\mathrm{C}_{43} \mathrm{H}_{54} \mathrm{O}_{13} \mathrm{SiNa}(\mathrm{M}+\mathrm{Na})$ 829.3226; found: 829.3221.

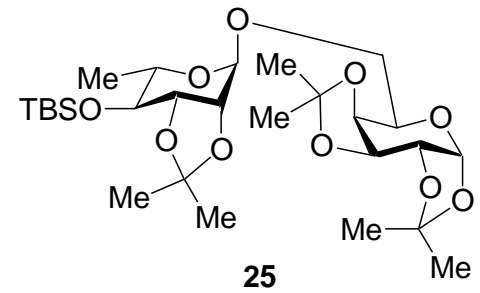

$\mathbf{R}_{\mathbf{f}}=0.37$ (hexanes/ethyl acetate, 6/1)

${ }^{1} \mathbf{H}$ NMR (CDCl 3 , 500 MHz): $\delta=5.51$ (d, $\left.J=5 \mathrm{~Hz}, 1 \mathrm{H}\right), 4.96$ (s, 1H), 4.58 (dd, $J=8,2.5 \mathrm{~Hz}$, $1 \mathrm{H}), 4.29$ (dd, $J=5,2.5 \mathrm{~Hz}, 1 \mathrm{H}), 4.24$ (dd, $J=7.5,1.5 \mathrm{~Hz}, 1 \mathrm{H}), 4.13$ (d, $J=5.5 \mathrm{~Hz}, 1 \mathrm{H}$ ), 3.95 (t, $J=6.5 \mathrm{~Hz}, 1 \mathrm{H}), 3.84$ (dd, $J=10,6 \mathrm{~Hz}, 1 \mathrm{H}), 3.63-3.56(\mathrm{~m}, 2 \mathrm{H}), 3.29$ (dd, $J=9.5,7 \mathrm{~Hz}, 1 \mathrm{H})$, 1.52 (s, 3H), 1.49 (s, 3H), 1.43 (s, 3H), 1.31 (s, 9H), 1.19 (d, $J=6.5 \mathrm{~Hz}, 3 \mathrm{H}), 0.86$ (s, 9H), 0.12 (s, 3H), 0.06 (s, 3H).

${ }^{13} \mathbf{C}$ NMR (CDCl 3,125 MHz): $\delta=109.2,108.9,108.5,97.0,96.3,79.1,76.0,75.9,71.0,70.6$, 66.5, 65.9, 65.3, 28.1, 26.4, 26.1, 26.0, 25.9, 24.9, 24.3, 17.6, -4.0, -4.9.

IR (film, $\mathbf{c m}^{-1}$ ): $v=2934,1382$.

HRMS (ESI): calc. for $\mathrm{C}_{27} \mathrm{H}_{48} \mathrm{O}_{10} \mathrm{SiNa}(\mathrm{M}+\mathrm{Na})$ 583.2909; found: 583.2927.

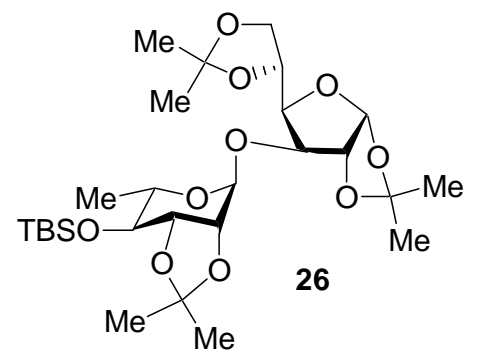

$\mathbf{R}_{\mathbf{f}}=0.34$ (hexanes/ethyl acetate, 6/1)

${ }^{1} \mathbf{H}$ NMR (CDCl 3,500 MHz): $\delta=5.86$ (d, $\left.J=3.5 \mathrm{~Hz}, 1 \mathrm{H}\right), 5.08$ (s, 1H), 4.53 (d, $J=3.5 \mathrm{~Hz}$, $1 \mathrm{H}), 4.40$ (d, $J=3.5 \mathrm{~Hz}, 1 \mathrm{H}), 4.26-4.23(\mathrm{~m}, 1 \mathrm{H}), 4.11$ (dd, $J=8.5,6.5 \mathrm{~Hz}, 1 \mathrm{H}), 4.08$ (dd, $J=$ 8.5, $3 \mathrm{~Hz}, 1 \mathrm{H}$ ), 4.05 (d, $J=5.5 \mathrm{~Hz}, 1 \mathrm{H}), 3.93-3.87$ (m, 2H), 3.84 (dd, $J=10,6 \mathrm{~Hz}, 1 \mathrm{H}), 3.30$ (dd, $J=10,7 \mathrm{~Hz}, 1 \mathrm{H}), 1.50$ (s, 3H), 1.49 (s, 3H), 1.38 (s, 3H), 1.32 (s, 3H), 1.29 (s, 6H), 1.16 (d, $J=$ $6.5 \mathrm{~Hz}, 3 \mathrm{H}), 0.85$ (s, 9H), 0.11 (s, 3H), 0.05 (s, 3H).

${ }^{13} \mathbf{C}$ NMR (CDCl 3 , $\left.125 \mathbf{M H z}\right): \delta=112.0,109.1,109.0,105.3,94.6,81.7,81.0,79.2,76.1,76.0$, 75.5, 71.7, 68.0, 66.4, 28.1, 26.74, 26.69, 26.4, 26.1, 25.8, 25.1, 17.4, -3.9, -5.0.

IR (film, $\mathbf{c m}^{-\mathbf{1}}$ ): $v=2934,1381,1372,1078$.

HRMS (ESI): calc. for $\mathrm{C}_{27} \mathrm{H}_{48} \mathrm{O}_{10} \mathrm{SiNa}(\mathrm{M}+\mathrm{Na})$ 583.2909; found: 583.2918. 


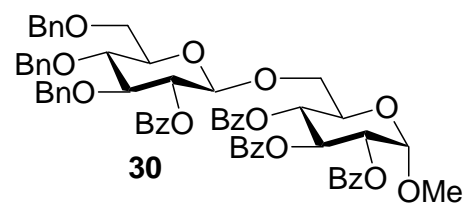

The synthesis of disaccharide $\mathbf{3 0}$ has been reported. ${ }^{\text {vii }}$

${ }^{1} \mathbf{H}$ NMR (CDCl 3 , 500 MHz): $\delta=8.05-7.78(\mathrm{~m}, 8 \mathrm{H}), 7.56-7.18(\mathrm{~m}, 27 \mathrm{H}), 6.05$ (t, $J=10.0 \mathrm{~Hz}$, $1 \mathrm{H}$ ), 5.30 (t, $J=9.0 \mathrm{~Hz}, 1 \mathrm{H}), 5.27$ (d, $J=7.0 \mathrm{~Hz}, 1 \mathrm{H}), 5.06$ (dd, $J=10.0,3.5 \mathrm{~Hz}, 1 \mathrm{H}), 4.85$ (d, $J$ $=3.5 \mathrm{~Hz}, 1 \mathrm{H}), 4.80(\mathrm{~d}, J=11.0 \mathrm{~Hz}, 1 \mathrm{H}), 4.73(\mathrm{~d}, J=11.0 \mathrm{~Hz}, 1 \mathrm{H}), 4.67(\mathrm{~d}, J=11.0 \mathrm{~Hz}, 1 \mathrm{H})$, 4.61-4.52 (m, 3H), 4.47 (d, $J=12.0 \mathrm{~Hz}, 1 \mathrm{H}), 4.17$ (ddd, $J=10.0,8.5,1.5 \mathrm{~Hz}, 1 \mathrm{H}), 4.07$ (dd, $J=$ 11.0, $1.5 \mathrm{~Hz}, 1 \mathrm{H}$ ), 3.84 (d, $J=9.0 \mathrm{~Hz}, 1 \mathrm{H}), 3.77$ (t, $J=9.0 \mathrm{~Hz}, 1 \mathrm{H}), 3.73-3.68$ (m, 2H), 3.64 (dd, $J=11.0,8.0 \mathrm{~Hz}, 1 \mathrm{H}), 3.52$ (dd, $J=9.5,5.5,3.5 \mathrm{~Hz}, 1 \mathrm{H}), 3.01$ (s, $3 \mathrm{H})$.

${ }^{13} \mathbf{C}$ NMR (CDCl 3,125 MHz): $\delta=165.71,165.7,165.5,165.3,133.4,133.3,133.1,133.0$, 129.9, 129.8, 129.6, 128.4, 128.3, 128.25, 128.2, 127.9, 127.8, 127.7, 127.63, 127.6, 101.7, 96.3, 82.7, 77.9, 77.3, 77.0, 76.8, 75.3, 75.1, 75.0, 73.7, 73.5, 72.0, 70.5, 69.7, 68.9, 68.7, 68.4, 54.9.

IR (film, $\mathbf{c m}^{-1}$ ): $v=2936,1728,1452,1094,1069,1027,735$.

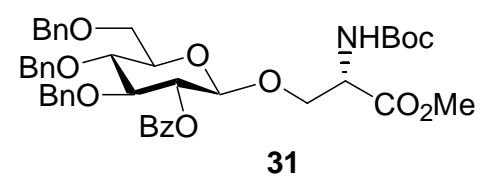

${ }^{1}$ H NMR (CDCl 3 , 500 MHz): $\delta=8.02$ (d, $\left.J=7.5 \mathrm{~Hz}, 2 \mathrm{H}\right), 7.58-7.11(\mathrm{~m}, 18 \mathrm{H}), 5.28$ (d, $J=8.0$ Hz, 1H), 5.22 (t, $J=8.5 \mathrm{~Hz}, 1 \mathrm{H}), 4.80$ (d, $J=10.5 \mathrm{~Hz}, 1 \mathrm{H}), 4.73$ (d, $J=11.5 \mathrm{~Hz}, 1 \mathrm{H}), 4.65$ (d, $J$ $=11.0 \mathrm{~Hz}, 1 \mathrm{H}), 4.63(\mathrm{~d}, J=12.0 \mathrm{~Hz}, 1 \mathrm{H}), 4.56(\mathrm{dd}, J=10.5,4.5 \mathrm{~Hz}, 1 \mathrm{H}), 4.52(\mathrm{~d}, J=8.0 \mathrm{~Hz}$, 1H), 4.34 (dd, $J=8.0,3.5$ Hz, 1H), 4.24 (d, $J=10.0$ Hz, 1H), 3.81-3.77 (m, 3H), 3.74 (d, $J=3.0$ $\mathrm{Hz}, 2 \mathrm{H}), 3.03$ (s, 3H), 3.36 (bs, 1H), 1.36 (s, 9H).

${ }^{13} \mathbf{C}$ NMR (CDCl 3 , 125 MHz): $\delta=170.5,165.2,155.4,137.9,137.8,137.7,133.2,129.8,128.4$, 128.3, 128.0, 127.9, 127.8, 127.72, 127.7, 101.2, 82.5, 77.7, 75.3, 75.1, 74.9, 73.6, 73.4, 68.9, 68.5, 53.9, 52.5, 28.3.

IR (film, $\mathbf{c m}^{-1}$ ): $v=3437,3353,3296,2951,2870,1723,1601,1497,1453,1365,1268,1161$, 1094, 1070, 826, 737, 712, 699.

HRMS (ESI): calc. for $\mathrm{C}_{43} \mathrm{H}_{49} \mathrm{NO}_{11} \mathrm{Na}[\mathrm{M}+\mathrm{Na}]$ 778.3203; found: 778.3224 .

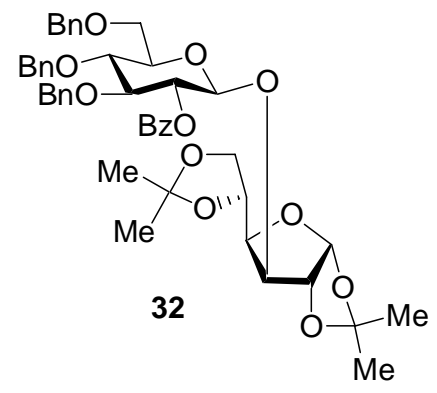

${ }^{1} \mathbf{H}$ NMR (CDCl, 500 MHz): $\delta=7.97$ (d, $\left.J=7.5 \mathrm{~Hz}, 2 \mathrm{H}\right), 7.58-7.11$ (m, 18H), 5.35 (d, $J=4.0$ Hz, 1H), 5.19 (t, $J=8.5 \mathrm{~Hz}, 1 \mathrm{H}), 4.80$ (d, $J=11.0 \mathrm{~Hz}, 1 \mathrm{H}), 4.73$ (d, $J=11.0 \mathrm{~Hz}, 1 \mathrm{H}), 4.65-4.54$ (m, 5H), 4.36 (dd, $J=11.0,6.0 \mathrm{~Hz}, 1 \mathrm{H}), 4.26-4.22$ (m, 3H), 4.02 (dd, $J=9.0,7.0 \mathrm{~Hz}, 1 \mathrm{H}$ ), 3.93 (dd, $J=9.0,6.0 \mathrm{~Hz}, 1 \mathrm{H}$ ), 3.81-3.75 (m, 2H), 3.73 (d, $J=3.0 \mathrm{~Hz}, 2 \mathrm{H}), 3.54-3.53(\mathrm{~m}, 1 \mathrm{H}), 1.39$ (s, 3H), 1.36 (s, 3H), 1.25 (s, 3H), 1.08 (s, 3H). 
${ }^{13} \mathbf{C}$ NMR (CDCl 3,125 MHz): $\delta=164.8,138.0,137.8,137.7,133.4,129.6,128.53,128.5$, 128.4, 128.3, 128.1, 127.97, 127.9, 127.8, 127.72, 127.7, 111.8, 108.5, 104.9, 99.7, 82.7, 82.3, 80.7, 80.4, 77.8, 75.7, 75.1, 74.8, 73.7, 73.6, 73.2, 68.5, 65.9.

IR (film, $\mathbf{c m}^{-1}$ ): $v=2987,2935,1723,1600,1453,1372,1266,1071,1026,826,712,698$.

HRMS (ESI): calc. for $\mathrm{C}_{46} \mathrm{H}_{52} \mathrm{O}_{12} \mathrm{Na}[\mathrm{M}+\mathrm{Na}]$ 819.3356; found: 819.3347.

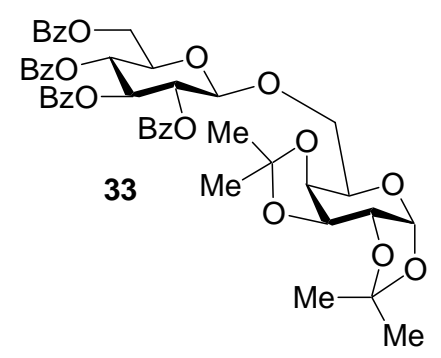

The synthesis of disaccharide $\mathbf{3} 3$ has been reported. ${ }^{\text {viii }}$

${ }^{1} \mathbf{H}$ NMR (CDCl, $\left.500 \mathrm{MHz}\right): \delta=8.01(\mathrm{~d}, J=8.0 \mathrm{~Hz}, 2 \mathrm{H}), 7.95(\mathrm{~d}, J=8.0 \mathrm{~Hz}, 2 \mathrm{H}), 7.88(\mathrm{~d}, J=$ $8.0 \mathrm{~Hz}, 2 \mathrm{H}), 7.80$ (d, $J=8.0 \mathrm{~Hz}, 2 \mathrm{H}), 7.53-7.24(\mathrm{~m}, 12 \mathrm{H}), 5.88(\mathrm{t}, J=9.5 \mathrm{~Hz}, 1 \mathrm{H}), 5.65(\mathrm{t}, J=$ $9.5 \mathrm{~Hz}, 1 \mathrm{H}$ ), 5.51 (dd, $J=9.5,8.0 \mathrm{~Hz}, 1 \mathrm{H}), 5.40$ (d, $J=5.0 \mathrm{~Hz}, 1 \mathrm{H}), 5.01$ (d, $J=8.0 \mathrm{~Hz}, 1 \mathrm{H}$ ), 4.62 (dd, $J=12.0,3.0 \mathrm{~Hz}, 1 \mathrm{H}), 4.46$ (dd, $J=12.0,5.5 \mathrm{~Hz}, 1 \mathrm{H}), 4.40$ (dd, $J=8.0,2.5 \mathrm{~Hz}, 1 \mathrm{H}$ ), 4.18 (dd, $J=5.0,2.5 \mathrm{~Hz}, 1 \mathrm{H}$ ), 4.15 (ddd, $J=8.5,5.0,3.0 \mathrm{~Hz}, 1 \mathrm{H}$ ), 4.07 (dd, $J=8.0,1.5 \mathrm{~Hz}, 1 \mathrm{H}$ ), 4.00 (dd, $J=10.5,3.5 \mathrm{~Hz}, 1 \mathrm{H}$ ), 3.88-3.81 (m, 2H), 1.35 (s, 3H), 1.22, (s, 3H), 1.18 (s, 3H), 1.17 (s, 3H).

${ }^{13} \mathbf{C ~ N M R ~ ( C D C l}_{3}, 125$ MHz): $\delta=166.1,165.8,165.2,165.1,133.4,133.1,133.0,130.0,129.8$, 129.7, 129.4, 128.9, 128.8, 128.7, 128.4, 128.3, 128.24, 128.2, 109.3, 108.4, 101.2, 96.2, 73.0, 72.2, 71.8, 70.9, 70.5, 70.4, 69.8, 68.3, 67.5, 63.2, 25.9, 25.7, 24.8, 24.2.

IR (film, $\mathbf{c m}^{-1}$ ): $v=1730,1601,1452,1374,1266,1094,1069,1026,825,709$.

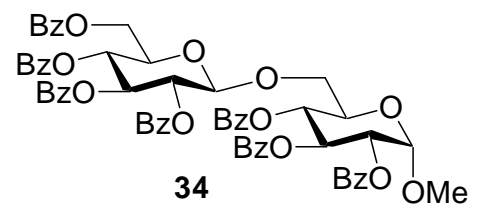

The synthesis of disaccharide $\mathbf{3 4}$ has been reported. ${ }^{\text {ix }}$

${ }^{1} \mathbf{H}$ NMR (CDCl 3 , 500 MHz): $\delta=7.96$ (dd, $\left.J=8.0,7.0 \mathrm{~Hz}, 4 \mathrm{H}\right), 7.90$ (d, $\left.J=7.0 \mathrm{~Hz}, 2 \mathrm{H}\right), 7.87$ (d, $J=7.5 \mathrm{~Hz}, 2 \mathrm{H}), 7.84$ (d, $J=7.5 \mathrm{~Hz}, 2 \mathrm{H}), 7.80$ (d, $J=7.5 \mathrm{~Hz}, 2 \mathrm{H}), 7.77$ (d, $J=7.5 \mathrm{~Hz}, 2 \mathrm{H}$ ), 7.53-7.20 (m, 16H), $6.04(\mathrm{t}, J=10.0 \mathrm{~Hz}, 1 \mathrm{H}), 5.89$ (t, $J=10.0 \mathrm{~Hz}, 1 \mathrm{H}), 5.63$ (t, $J=10.0 \mathrm{~Hz}$, $1 \mathrm{H}), 5.54$ (d, $J=9.5,7.5 \mathrm{~Hz}, 1 \mathrm{H}), 5.29$ (t, $J=9.5 \mathrm{~Hz}, 1 \mathrm{H}), 5.06$ (dd, $J=10.0,2.5 \mathrm{~Hz}, 1 \mathrm{H}), 4.95$ (d, $J=8.0 \mathrm{~Hz}, 1 \mathrm{H}), 4.91$ (d, $J=4.0 \mathrm{~Hz}, 1 \mathrm{H}), 4.58$ (dd, $J=12.5,3.0 \mathrm{~Hz}, 1 \mathrm{H}), 4.42$ (dd, $J=12.0$, $5.0 \mathrm{~Hz}, 1 \mathrm{H}), 4.19$ (t, $J=8.0 \mathrm{~Hz}, 1 \mathrm{H}), 4.12$ (dd, $J=9.5,4.0 \mathrm{~Hz}, 1 \mathrm{H}), 4.08$ (d, $J=11.0 \mathrm{~Hz}, 1 \mathrm{H})$, 3.76 (dd, $J=11.5,7.5 \mathrm{~Hz}, 1 \mathrm{H}), 3.07$ (s, 3H).

${ }^{13} \mathbf{C}$ NMR (CDCl 3 , 125 MHz): $\delta=166.1,165.8,165.6,165.4,165.2,133.4,133.3,133.2,133.1$, 133.0, 129.8, 129.7, 129.6, 129.3, 129.2, 129.0, 128.7, 128.4, 128.31, 128.3, 128.2, 101.7, 96.4, 72.8, 72.3, 71.9, 71.8, 70.3, 69.6, 68.9, 68.7, 63.0, 55.0.

IR (film, $\mathbf{c m}^{-1}$ ): $v=2929,1729,1601,1451,1315,1267,1108,1094,1069,1026,911,708$. 


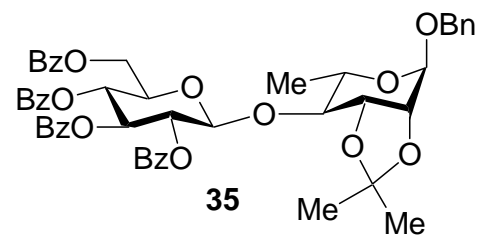

${ }^{1} \mathbf{H}_{\text {NMR }}\left(\mathbf{C D C l}_{3}, \mathbf{5 0 0} \mathbf{M H z}\right): \delta=8.07-7.81(\mathrm{~m}, 7 \mathrm{H}), 7.53-7.17(\mathrm{~m}, 18 \mathrm{H}), 5.89$ (t, $J=10.0 \mathrm{~Hz}$, 1H), 5.62 (t, $J=10.0 \mathrm{~Hz}, 1 \mathrm{H}$ ), 5.46 (dd, $J=10.0,8.0 \mathrm{~Hz}, 1 \mathrm{H}$ ), 5.31 (d, $J=8.0 \mathrm{~Hz}, 1 \mathrm{H}$ ), 4.96 (s, 1H), 4.65 (dd, $J=5.5,2.5 \mathrm{~Hz}, 1 \mathrm{H}$ ), 4.60 (d, $J=12.0 \mathrm{~Hz}, 1 \mathrm{H}$ ), 4.46 (d, $J=5.5 \mathrm{~Hz}, 1 \mathrm{H}$ ), 4.41 (d, $J$ $=11.5 \mathrm{~Hz}, 1 \mathrm{H}), 4.11(\mathrm{ddd}, J=9.5,5.5,3.5 \mathrm{~Hz}, 1 \mathrm{H}), 4.01-3.96(\mathrm{~m}, 2 \mathrm{H}), 3.63(\mathrm{dq}, J=9.5,6.0 \mathrm{~Hz}$, $1 \mathrm{H}$ ), 3.56 (dd, $J=10.0,6.5 \mathrm{~Hz}, 1 \mathrm{H}), 1.45$ (s, 3H), 1.26 (d, $J=6.0 \mathrm{~Hz}, 3 \mathrm{H}), 1.23$ (s, 3H).

${ }^{13} \mathbf{C}$ NMR (CDCl 3 , 125 MHz): $\delta=166.1,165.8,165.3,136.9,133.6,133.2,133.1,130.0,129.9$, 129.8, 129.76, 129.7, 129.6, 129.5, 128.9, 128.8, 128.5, 128.4, 128.3, 128.2, 127.9, 109.2, 100.4, 95.9, 80.7, 78.0, 76.0, 73.1, 72.3, 72.1, 70.0, 69.1, 64.0, 63.2, 29.7, 27.9, 26.2, 17.5.

IR (film, $\left.\mathbf{c m}^{-1}\right): v=2983,2933,1732,1601,1452,1380,1266,1177,1106,1092,1069,1026$, 733, 709, 686.

HRMS (ESI): calc. for $\mathrm{C}_{50} \mathrm{H}_{48} \mathrm{O}_{14} \mathrm{Na}[\mathrm{M}+\mathrm{Na}]$ 895.2942; found: 895.2929.

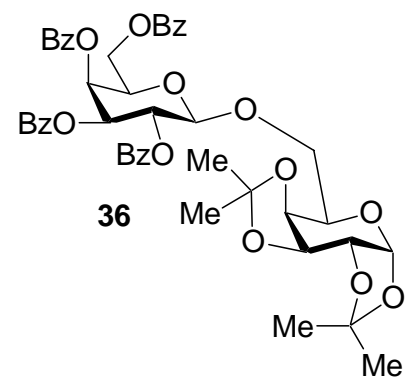

$\mathbf{R}_{\mathbf{f}}=0.38$ (9/1, Benzene/Ethyl Acetate)

${ }^{1} \mathbf{H}$ NMR (CDCl 3,500 MHz): $\delta=8.07(\mathrm{~d}, J=7.5 \mathrm{~Hz}, 2 \mathrm{H}), 8.01(\mathrm{~d}, J=7.5 \mathrm{~Hz}, 2 \mathrm{H}), 7.96(\mathrm{~d}, J=$ 7.5, 2H), 7.76 (d, $J=7.5 \mathrm{~Hz}, 2 \mathrm{H}), 7.59$ (t, $J=7 \mathrm{~Hz}, 1 \mathrm{H}), 7.54$ (t, $J=7.5 \mathrm{~Hz}, 1 \mathrm{H}), 7.46(\mathrm{~m}, 3 \mathrm{H})$, 7.41 (t, $J=8 \mathrm{~Hz}, 3 \mathrm{H}) 7.34$ (t, $J=7.5 \mathrm{~Hz}, 2 \mathrm{H}), 7.22$ (m, 3H), 5.97 (d, IH, $J=2.5 \mathrm{~Hz}), 5.79$ (t, $J=$ $10 \mathrm{~Hz}, 1 \mathrm{H}$ ), 5.59 (dd, $J=10.0,3.0 \mathrm{~Hz}, 1 \mathrm{H}), 5.40$ (d, $J=4 \mathrm{~Hz}, 1 \mathrm{H}), 5.0(\mathrm{~d}, J=8 \mathrm{~Hz}, 1 \mathrm{H}), 4.65$ (dd, $J=11.0,6.5 \mathrm{~Hz}, 1 \mathrm{H}$ ), 4.40 (m. 2H), 4.32 (t, $J=6.6 \mathrm{~Hz}, 1 \mathrm{H}$ ), 4.19 (d, $1 \mathrm{H}, J=2.5 \mathrm{~Hz}$ ), (m, $1 \mathrm{H}), 4.04(\mathrm{~m}, 1 \mathrm{H}), 3.89(\mathrm{~m}, 2 \mathrm{H}), 1.37$ (s, 3H), 1.22 (s, 3H), 1.20 (s, 3H), 1.18 (s, 3H).

${ }^{13} \mathbf{C}_{\text {NMR }}\left(\mathbf{C D C l}_{3}, 125 \mathrm{MHz}\right): 166.0,165.6,165.5,165.3,133.5,133.2,133.0,129.9,129.8$, 128.6, 128.4, 128.3, 128.2, 109.3, 108.4, 101.7, 96.2, 71.8, 71.3, 71.0, 70.5, 70.3, 69.7, 68.4, 68.2, 67.4, 62.0, 25.9, 25.7, 24.8, 24.2.

IR (film, $\mathbf{c m}^{-1}$ ): $v$ = 3065, 2985, 2935, 1727, 1605, 1453, 1381, 1267, 1103, 1069.

HRMS (ESI): calc. for $\mathrm{C}_{46} \mathrm{H}_{46} \mathrm{O}_{15} \mathrm{Na}$ [M+Na] 861.2734; found: 895.2766. 


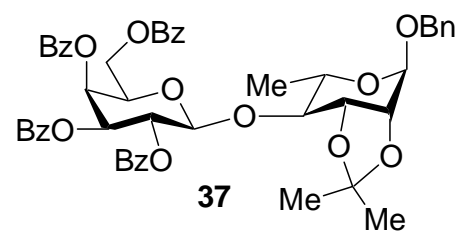

$\mathbf{R}_{\mathbf{f}}=0.29$ (25/1,Benzene/Ethyl acetate)

${ }^{1} \mathbf{H}$ NMR (CDCl 3,500 MHz): $\delta=8.06$ (d, $\left.J=7 \mathrm{~Hz}, 2 \mathrm{H}\right), 8.00$ (dd, $\left.J=7,4 \mathrm{~Hz}, 4 \mathrm{H}\right), 7.78$ (d, $J=7$ $\mathrm{Hz}, 2 \mathrm{H}), 7.61(\mathrm{t}, J=7.5 \mathrm{~Hz} 1 \mathrm{H}), 7.54(\mathrm{t}, J=7.5 \mathrm{~Hz}, 1 \mathrm{H}), 7.48(\mathrm{t},=7.5 \mathrm{~Hz}, 3 \mathrm{H}), 7.41(\mathrm{t}, J=$ $7.5 \mathrm{~Hz}, 3 \mathrm{H}), 7.35$ (m, 9H), $7.31(\mathrm{t}, J=6 \mathrm{~Hz}, 2 \mathrm{H}), 7.26(\mathrm{~m}, 3 \mathrm{H}), 5.96$ (d, $J=2.5 \mathrm{~Hz}, 1 \mathrm{H}), 5.71$ (t, $J=10.5 \mathrm{~Hz}, 1 \mathrm{H}$ ), 5.59 (dd, $\mathrm{J}=10.5,3.5 \mathrm{~Hz}, 1 \mathrm{H}), 5.23$ (d, $J=8 \mathrm{~Hz}, 1 \mathrm{H}) 4.98$ (s, 1H), 4.62 (m, 2H), $4.42(\mathrm{~m}, 2 \mathrm{H}), 4.29(\mathrm{t}, J=6 \mathrm{~Hz}, 1 \mathrm{H}), 4.01(\mathrm{~s}, 1 \mathrm{H}), 3.99(\mathrm{~m}, 1 \mathrm{H}), 3.70(\mathrm{~m}, 1 \mathrm{H}), 3.58(\mathrm{~m}, 1 \mathrm{H})$, 1.46 (s, 3H), 1.34 (d, $J=6.5 \mathrm{~Hz}, 3 \mathrm{H}), 1.21$ (s, 3H).

${ }^{13}$ C NMR (CDCl 3 , 125 MHz): 165.6, 133.5, 133.3, 133.2, 133.1, 129.9, 128.6, 128.5, 128.4, 128.3, 128.0, 101.2, 96.0, 81.5, 78.0, 76.0, 71.9, 71.4, 70.0, 69.1, 68.3, 64.1, 62.2, 27.9, 26.1, 17.6.

IR (film, $\mathbf{c m}^{-\mathbf{1}}$ ): $v=$ 3063, 3033, 2964, 2935, 1730, 1602, 1450, 1261, 1095, 1068.

HRMS (ESI): calc. for $\mathrm{C}_{50} \mathrm{H}_{48} \mathrm{O}_{14} \mathrm{Na}$ [M+Na] 895.2942; found: 895.2929.

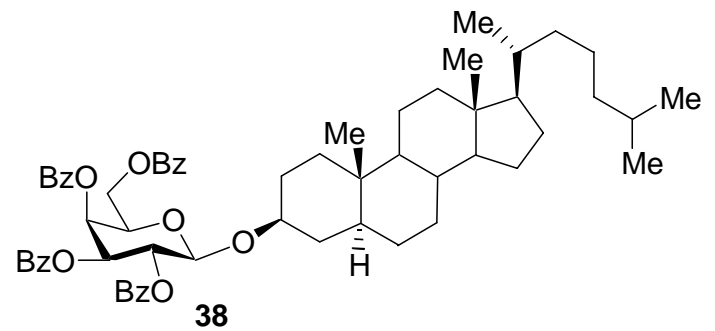

$\mathbf{R}_{\mathbf{f}}=\mathbf{0 . 3 6}$ (40/1 Benzene/Ethyl Acetate)

${ }^{1}$ HNMR (CDCl 3 , 500 MHz): $\delta=8.09(\mathrm{~d}, J=7.5 \mathrm{~Hz}, 2 \mathrm{H}), 8.01(\mathrm{~d}, J=7.5 \mathrm{~Hz}, 2 \mathrm{H}), 7.94(\mathrm{~d}, J=$ $7.0 \mathrm{~Hz}, 2 \mathrm{H}), 7.77(\mathrm{~d}, J=7.5 \mathrm{~Hz}, 2 \mathrm{H}), 7.59$ (t, $J=7.5 \mathrm{~Hz}, 1 \mathrm{H}), 7.55$ (t, $J=7.5 \mathrm{~Hz}, 1 \mathrm{H}), 7.49$ (m, $3 \mathrm{H}), 7.42(\mathrm{t}, J=10 \mathrm{~Hz}, 3 \mathrm{H}), 7.36(\mathrm{~m}, 3 \mathrm{H}), 7.22(\mathrm{~m}, 2 \mathrm{H}), 5.96(\mathrm{~d}, J=2.5 \mathrm{~Hz}, 1 \mathrm{H}), 5.74(\mathrm{t}, J=$ $10.5 \mathrm{~Hz}, 1 \mathrm{H}), 5.56$ (dd, $J=10.5,3.5 \mathrm{~Hz}, 1 \mathrm{H}), 4.86$ (d, $J=8 \mathrm{~Hz}, 1 \mathrm{H}), 4.66$ (dd, $J=11.5,7 \mathrm{~Hz}$, $1 \mathrm{H}), 4.41$ (dd, $J=11,6.5 \mathrm{~Hz}, 1 \mathrm{H}), 4.30$ (t, $J=6.5 \mathrm{~Hz}, 1 \mathrm{H}), 3.61(\mathrm{~m}, 1 \mathrm{H}, \mathrm{H}), 2.0-0.5(\mathrm{~m}, 47 \mathrm{H})$.

${ }^{13} \mathbf{C}$ NMR (CDCl 3,125 MHz): 166.0, 165.7, 165.3, 165.2, 130.1, 129.8, 129.7, 128.8, 128.6, 128.4, 128.3, 128.2, 100.6, 80.5, 71.9, 71.2, 70.0, 68.2, 62.1, 56.4, 56.3, 54.3, 44.7, 42.6, 40.0, 39.5, 36.9, 36.2, 35.8, 35.5, 35.4, 34.7, 32.0, 29.4, 28.6, 28.2, 28.0, 24.2, 23.8, 22.8, 22.6, 21.2, 18.7.

IR (film, $\mathbf{c m}^{-\mathbf{1}}$ ): $v$ =3067, 2935, 2863, 1730, 1602, 1450, 1266, 1106, 1068, 1027.

HRMS (ESI): Calc for $\mathrm{C}_{61} \mathrm{H}_{74} \mathrm{O}_{10} \mathrm{Na}[\mathrm{M}+\mathrm{Na}$ ] 989.5174; found: 989.5133 .

\footnotetext{
${ }^{\text {i }}$ Chreiten, F.; Chapleur, Y.; Castro, B.; Gross, B. J. Chem. Soc.; Perkin Trans. 1 1980, 381-384.

ii Dasgupta, F.; Garegg, P. Carbohydr. Res. 1990, 202, 225-238.

iii Fraser-Reid, B.; Konradsson, P.; Mootoo, D. R.; Udodong, U. J. Chem. Soc., Chem. Commun., 1988, 823-825.

iv (a) Kim, K. S.; Lee, Y. J.; Kim, H. Y.; Kang, S. S.; Kwon, S. Y. Org. Biomol. Chem. 2004, 2, 2408. (b) Houdier, S.; Vottero, P. J. A. Carbohydr. Res. 1992, 232, 349.

${ }^{v}$ Kim, K. S.; Lee, Y. J.; Kim, H. Y.; Kang, S. S.; Kwon, S. Y. Org. Biomol. Chem. 2004, 2, 2408.

${ }^{v i}$ Mukaiyama, T.; Matsubara, K.; Hora, M. Synthesis 1994, 1368.

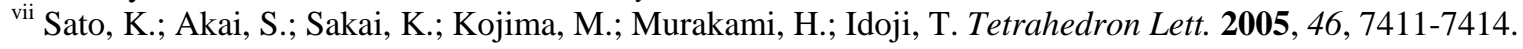


viii Gareg, P. J.; Norberg, J. Acta. Chemi. Scad. 1979, 116-118.

${ }^{\text {ix }}$ Guthrie, R. D.; Jenkins, A. D.; Roberts, G. A. F. J. Chem. Soc., Perkin. Trans. 1 1973, 2414-2417. 